\title{
PHILOSOPHIE DE LA NATURE ET COMMUNAUTÉ CHEZ SHAFTESBURY ET TOLAND
}

\author{
Fabienne BrUGÈRE
}

\begin{abstract}
Résumé : La publication par John Toland de l'Enquête sur la vertu ou le mérite de Shaftesbury semble indiquer une complicité, voire même une convergence de doctrine entre les deux penseurs. Or, cette convergence apparente masque la spécificité de chacun concernant surtout les concepts de nature et de communauté. Plus précisément, l'orientation de la nature par rapport à la communauté humaine ne se fait pas pour les mêmes raisons. Alors que Shaftesbury inclut nature et communauté dans une même perspective pour aboutir à l'analyse du lien social, Toland forge un projet de connaissance théorique de la nature qui l'affranchit de la définition essentiellement pratique de toute communauté humaine en général.
\end{abstract}

Mots clés : déisme, organisation de la nature, communauté humaine et société idéale, apprentissage social du monde.

Summary : When John Toland publishes Shaftesbury's book, An Inquiry Concerning Virtue or Merit, there seems to exist a complicity or even a doctrinal similarity between the two thinkers. Now, this apparent affinity hides the specificity of each author concerning the concepts of nature and community. More exactly, the orientation of nature with regard to the human community is not realized for the same reasons. When Shaftesbury includes nature and community in a same view in order to build the analysis of the social relations, Toland elaborates a theoretical knowledge of nature which allows him to avoid a practical definition of any human community in general.

Keywords : deism, organization of nature, human community and ideal society, social apprenticeship of the world.

Revue de synthèse : $4^{e} \mathrm{~S} . \mathrm{N}^{\mathrm{os}}$ 2-3, avr.-sept. 1995, p. 303-314. 
Zusammenfassung: John Tolands Edition von Shaftesburys An Inquiry Concerning Virtue or Merit scheint zu zeigen, daß die beiden Denker in ihren Ansichten in hohem Maße übereinstimmten. Hinter dieser vordergründigen Übereinstimmung verbergen sich jedoch ganz unterschiedliche Auffassungen von der Natur und der menschlichen Gemeinschaft. Genauer gesagt, ist die Natur bei den beiden nicht aus denselben Gründen auf die menschliche Gemeinschaft ausgerichtet. Während für Shaftesbury Natur und Gemeinschaft zusammen zu sozialen Beziehungen führen, erarbeitet Toland ein theoretisches Naturwissen, daß es ihm erlaubt, auf eine praktische Definition dessen, was ganz allgemein unter menschlicher Gemeinschaft zu verstehen ist, zu verzichten.

Stichwörter: Deismus, Aufbau der Natur, menschliche Gemeinschaft und ideale Gesellschaft, Sozialisierung.

Fabienne BruGèRE, née en 1965, est ancienne élève de l'École normale supérieure, et agrégée de philosophie. Elle enseigne à l'université de Brest et travaille sur la philosophie anglaise autour de 1700 , en particulier dans les domaines esthétique, moral et politique.

Adresse : Université de Bretagne occidentale, U.F.R. de lettres et sciences sociales, 20 rue Duquesne, site 3, BP 814, 29285 Brest Cedex. 
En 1699, John Toland publie le premier traité de Shaftesbury, l'Enquête sur la vertu ou le mérite, vraisemblablement à l'insu de l'auteur ${ }^{1}$; jusqu'en 1703, il reçoit aussi de Shaftesbury une pension. Au-delà du caractère fortuit de cette entente, une telle rencontre semble mettre à jour une option philosophique commune précisée dans l'Enquête, dans certains textes de Toland, relative au principe organisateur de la nature.

De fait, les quatrième et cinquième Lettres à Serena de John Toland s'assimilent à une véritable recherche cosmologique sur la nature : toutes les parties de l'univers se situent dans un mouvement constant de destruction et de création. L'activité de la nature, propre aux phénomènes naturels, se libère de toute fondation théologique. Or, apparemment, l'Enquête fait appel à une même perspective : la prévalence cosmologique au début du texte aboutit à un principe naturel d'administration de l'univers répandu dans chaque chose en vue de l'harmonie du tout. L'organisation de la nature en monde se fait dans les deux cas en dehors de toute référence apologétique au texte biblique. Par là même, est rendu possible un certain déisme ${ }^{2}$ dans lequel l'administration du monde ne s'impose plus par un être divin transcendant découvert par la Révélation mais résulte d'une organisation naturelle, rationnelle et humanisée de la déité.

Cependant, cette convergence apparente masque des implications philosophiques différentes quant à l'utilisation du concept de nature. Les quatrième et cinquième Lettres à Serena n'interdisent pas la possibilité d'une philosophie de la nature comme projet d'une connaissance théorique de celle-ci à partir de données fournies par la nature elle-même. En revanche, chez Shaftesbury, l'idée de nature subit une inflexion qui empêche toute approche théorique de la nature : le gouvernement de l'univers se fait en vue du bien de celui-ci. La communauté humaine comme possibilité d'un ensemble de relations ayant pour fin la vertu, exprime la dimension la plus accomplie de ce bien. La nature est humanisée, socialisée, moralisée puisque la communauté comme collection, coopération d'individus en vue de la réalisation d'un but commun, ici le bien du monde, sert de modèle et de révélateur pour découvrir les lois de la nature. En bref, alors que Shaftes-

1. Pour Shaftesbury, le texte de 1699 n'était pas terminé. L'Enquête fut publiée sous sa forme définitive dans la première édition des Characteristics of Men, Manners, Opinions, Times, en 1711. Pour comparer les deux versions, consulter l'édition Wolfram BENDA pour la Standard Edition, Complete Works, II, 2, Stuttgart, Frommann, 1984. Le véritable titre de 1699 est : An Inquiry Concerning Virtue, in Two Discourses.

2. Chez John Stuart Mill, dans l'Autobiographie, Paris, Aubier, 1993, p. 60, les notes de John M. RoBSON donnent la définition suivante du déisme lorsque Mill s'interroge sur l'origine des choses : « le déisme consiste à croire que le Créateur se révèle par le truchement de la loi naturelle plutôt que par les miracles et l'Écriture. » 
bury inclut les idées de nature et de communauté dans un même ordre pratique aboutissant à l'analyse du lien social, Toland forge un projet de connaissance théorique de la nature dont la définition essentiellement pratique de toute communauté humaine en général est exclue; la réflexion sur la communauté s'affranchit de toute origine commune avec l'idée de nature pour constituer un ordre normatif des individus totalement autonome.

L'orientation d'une pensée de la nature par rapport à la communauté ne se fait pas de la même façon ni pour les mêmes raisons chez Shaftesbury et chez Toland. Avec Shaftesbury, la réflexion sur le principe naturel conduit à un déisme pratique dans lequel l'organisation de la nature en monde est élucidée par des liens de sympathie pour tous les êtres vivants, de vertu chez les hommes, la communauté humaine étant spontanément convoquée comme ce qui peut le mieux réaliser le naturel moral de la nature. Avec Toland, il s'agit de mettre au point une véritable philosophie de la nature par le projet d'un déisme tout d'abord théorique qui intègre l'idée de nature dans une discussion des thèses de Spinoza et de Newton sur la possibilité d'une différence entre nature active et passive.

Dans cette perspective, la distinction établie par A. O. Aldridge ${ }^{3}$ entre "déisme humaniste" et "déisme scientifique » peut donner un cadre conceptuel à ces deux lectures différentes du principe de la nature : la première expression prouve l'existence de la déité par des raisons morales alors que la seconde s'inspire de la méthode scientifique de Newton et professe la découverte de la déité grâce aux signes d'ordre et de combinaison qui se manifestent dans l'univers physique.

Choisir entre ces deux déismes suppose préalablement de développer, d'une part, la réflexion autonome sur l'idée de nature chez Toland qui décroche le social du naturel pour élaborer la communauté non plus sous forme d'impératif mais dans un cadre normatif propre et, d'autre part, le projet shaftesburien de la commensurabilité, voire même de l'identité du principe naturel du monde et de l'impératif communautaire. En d'autres termes, pourquoi la nature inclut-elle la communauté d'un côté et l'exclutelle de l'autre au risque, nous le verrons, d'écarter la notion de communauté comme réflexion sur les rapports sociaux au profit de la société idéale?

PHYSIQUE DE LA NATURE ET COMMUNAUTÉ IDÉALE CHEZ TOLAND

John Toland a sans doute lu l'Enquête comme un plaidoyer en faveur de la cohérence interne de la nature pour expliquer tous les êtres naturels par

3. Alfred Owen ALDRIDGE, "Shaftesbury and the Deist manifesto", Transactions of the American Philosophical Society, new series, vol. 41, 1951, p. 298. 
des lois d'organisation communes. Shaftesbury écrit au début de l'Enquête: «il y a [...] un système de toutes choses et une nature universelle ${ }^{4} ;$;'idée de nature est universellement vraie et nécessairement répandue sur tout ce qui se manifeste dans le monde. Le problème est alors de savoir quels sont les lois ou le principe que le terme de nature recouvre. Quel contenu Toland donne-t-il à ce qu'il croit reconnaître chez Shaftesbury comme l'idée d'une organisation sans faille de la nature?

La cinquième Lettre à Serena répond à cette question parce qu'elle ambitionne la découverte de la structure ${ }^{5}$ du monde, la mise à jour d'un principe constitutif, d'une cause simple d'explication de la manière d'être des phénomènes naturels. La structure ou le système du monde consiste en des lois de la nature, générales, simples et universelles auxquelles aucun être naturel ne fait exception. Le texte de la nature n'est jamais soumis aux mystères d'une création divine transcendante mais au contraire, il est totalement lisible pour la raison humaine qui connaît la cause ou le principe de ce qui se manifeste dans la réalité naturelle.

Pour Toland, le principe commun à tous les êtres naturels réside dans le mouvement, qu'il soit évident comme dans les corps fluides : circulation du sang et de la sève, ou caché dans les corps solides : pierres, métaux ${ }^{6}$.

La véritable définition du mouvement n'est pas celle du mouvement local comme passage d'un lieu à un autre qui décrit seulement le mouvement apparent ${ }^{7}$ mais l'activité inhérente aux corps eux-mêmes, cause de tout mouvement : par exemple, un corps apparemment au repos produit une action de résistance interne à des forces extérieures qui tendent à le détruire. L'activité des corps résulte d'une composition et d'un affrontement de forces répulsives et attractives.

Il faut ajouter que cette activité comme force, réside dans la matière ellemême. La philosophie de la nature comme théorie de la connaissance des êtres naturels est une physique de l'activité inhérente aux corps dans laquelle ces derniers sont définis comme des variations de matière ${ }^{8}$.

4. Shaftesbury, Characteristics of Men, Manners, Opinions, Times, Hildesheim/New York, Olms, 1978, repr. de l'éd. de Londres, 1711, vol. II, p. 20 : « there is [...] a System of all Things, and a Universal Nature. ”

5. John Toland, Letters to Serena, Letter V, repr. de l'éd. de Londres, 1704, Stuttgart, Frommann, 1964, p. 184 : «The Constitution and Fabric of the Universe », «The Fabric of the World. »

6. Ibid., p. 197 : «The Circulation of the Blood and Sap to every imaginable Part, are now no Secrets in Natural philosophy. » Cette description du principe des êtres naturels requiert le titre non anodin de philosophie naturelle.

7. J. Toland pense sans doute à un passage de John Locke dans l'Essai philosophique concernant l'entendement humain, Paris, Vrin, 1989, liv. III, chap. 4, \$ 9, p. 339 ; ceux qui définissent le mouvement comme « un passage d'un lieu dans un autre » ne font que donner un synonyme du mouvement mais ne définissent nullement le mot.

8. J. Toland, op. cit. supra n. 5, p. 173 : "On this occasion, to avoid all Ambiguity, 'tis convenient to inform you, that by Bodys I understand certain Modifications of Matter. " 
Comparer des corps revient à comparer des quantités de matière. La diversité dans la cohésion des corps (solides, fluides, vivants), dans leur situation temporelle et spatiale fournit chaque fois des preuves de l'activité inhérente à la matière.

Dès lors, le projet d'une philosophie naturelle comme mise à nu des structures du monde conduit à trouver les causes des mouvements des corps, le mouvement local n'étant que l'effet d'une combinaison de forces physiques. L'insistance sur l'activité de la matière et ceci, contre toute une tradition philosophique que Toland ne ménage pas, semble faire l'économie de tout principe spirituel d'activité et de toute spécificité humaine.

Par ailleurs, la mise en perspective de l'autonomie d'organisation de la matière s'assortit d'un réalisme de la connaissance qui affranchit l'analyse des corps de toute métaphysique ou de tout présupposé philosophique $a$ priori dans l'étude de la nature. Conformément à la tradition newtonienne des Principes mathématiques de la philosophie naturelle, la cinquième Lettre à Serena refuse les hypothèses indépendantes de l'expérience au profit de causes simples qui n'impliquent pas de divorce entre pensée et réalité ${ }^{9}$. La réflexion sur la nature évite le recours à une sorte d'imagination philosophique, la métaphysique; cette dernière enfante des fictions et non des vérités sur la nature parce qu'elle fait toujours régresser les causes à l'infini.

À la question : d'où vient le mouvement apparent? Toland se contente de répondre : d'une activité générale des corps matériels. La théorie de la nature s'induit de l'observation et de l'expérience des corps en mouvement. La philosophie naturelle n'est pas une métaphysique de la nature ni même une tentative de faire correspondre le spirituel et le matériel dans la connaissance du monde. Elle élabore une chaîne des êtres matériels sans discontinuité dans laquelle les relations horizontales passent par la destruction et l'engendrement ininterrompus ${ }^{30}$.

Les phénomènes naturels dans un mouvement sans fin de renouveau et d'usure sont tous régis par "la même action perpétuelle et universelle " Cette définition traduit la nécessité de la nature et établit l'ordonnancement, l'organisation du monde à partir de ses manifestations dans l'univers physique puisque l'activité est une qualité essentielle de la matière.

9. Alexandre KoYrÉ, Études newtoniennes, Paris, Gallimard, 1968, p. 60: «hypotheses non fingo ", « je ne feins pas d'hypothèses », veut dire tout simplement: « je n'utilise pas de fictions et de propositions fausses comme prémisses et explications. 》 Il faut bien sâr souligner que, sur d'autres points comme la conception de l'espace par exemple, Toland n'est pas newtonien.

10. J. Toland, op. cit. supra n. 5, p. 188.

11. Ibid., p. 237. 
Face à cette réduction physicaliste de l'idée de monde dans laquelle l'homme n'est qu'un corps en mouvement parmi d'autres, comment penser la notion de communauté sinon en tournant le dos à cette philosophie naturelle?

Il semblerait que la philosophie de Toland soit condamnée à se scinder entre, d'une part, l'approche théorique de la nature radicalement matérielle et réaliste et, d'autre part, l'élaboration d'une pensée du lien social qui ne peut ni s'intégrer dans l'approche physique du monde, ni se soumettre à l'universalité et à la nécessité. De fait, tout oppose la communauté comme collection d'individus en vue d'un horizon commun à la philosophie naturelle. Nous aimerions montrer que la communauté définit une appartenance en fonction de normes communes aux individus qu'ils n'ont pas posées eux-mêmes comme si le sens de la communauté leur échappait. Elle se réfère à d'autres lois d'organisation que celles qui entraînent la nécessité, la possibilité de faire correspondre vérité et réalité, la physique des corps.

La question de la communauté humaine est mise au point dans la première Lettre à Serena sur « l'origine et la force des préjugés », ces derniers constituant le ciment principal de notre appartenance déterminée à un groupe dont les règles nous sont imposées dès la naissance sans que nous puissions les choisir ou les juger. Les préjugés sont la trace de notre apprentissage social du monde; ils expriment la société dans laquelle nous vivons et avons été éduqués, imposés par l'autorité et cristallisés par nos passions. En ce sens, appartenir à une communauté revient à pérenniser des préjugés.

L'analyse du lien social dans la communauté à partir du préjugé discrédite toute crédibilité en une communauté réelle et ceci, pour plusieurs raisons qui vont à l'encontre des analyses philosophiques de la cinquième Lettre à Serena.

Tout d'abord, le préjugé est arbitraire : il dépend des mours du pays, des coutumes, des rites religieux ${ }^{12}$. Il oppose la communauté réelle à la nécessité naturelle.

Le préjugé, arbitraire et relatif, crée pourtant une « seconde nature " ${ }^{13}$, une réalité non plus matérielle mais sociale et faussement vraie parce qu'elle produit un imaginaire social semblable à celui d'une métaphysique de la nature. Cet imaginaire est enraciné dans des croyances collectives

12. Celui qui a la chance de partir étudier à l'étranger prend conscience de l'arbitraire et du caractère relatif des lois et de la religion de son pays, cf. J. TolaND, op. cit. supra $\mathrm{n}$. 5 , Letter One, p. 6: «We are made little wiser, tho much more vain and conceited in the universitys, especially abroad, where the Professors (right or wrong) must accommodate all things to the Laws and the Religion of the country. "

13. Ibid., p. 13. 
véhiculées par des mythes mais aussi des coutumes, des rites religieux et l'autorité des lois.

Dès lors, la physique des corps est remplacée par une physique des passions ${ }^{14}$ qui empêche l'exercice d'un jugement sain : les préjugés se forment déjà dans des impressions prénatales impliquant une préformation du tempérament qui conduit les êtres humains vers une fausse nécessité.

L'analyse de la communauté qui entoure chaque homme dès sa naissance aboutit à une critique de la communauté réelle ou du lien social ordinaire entre les hommes. La communauté humaine telle qu'elle existe hic et nunc est un mauvais modèle de société. Elle est mensonge et s'avère incompatible avec les règles de vérité universelles de la philosophie naturelle. Cependant, la philosophie de John Toland ne ménage-t-elle pas la possibilité d'une autre conception de la communauté en accord avec la cinquième lettre?

Dans cette perspective, la deuxième partie du Tetradymus, «Clidophorus or of the Exoteric and Esoteric Philosophy », peut être interprétée comme un manifeste pour une autre vision de la communauté. Le texte incite à la défense d'une communauté de raison contre une communauté de passion, la communauté de raison concernant le petit cercle de proches avec lesquels il est possible de partager une vérité sans danger d'une éventuelle censure ou incompréhension. La communauté de raison a pour souci la vérité théorique et un goût commun pour les démonstrations; c'est une communauté de philosophes, alors que la communauté au sens large est populaire et concerne le peuple en général guidé par l'opinion, c'est-à-dire les préjugés, la coutume et la religion révélée ${ }^{15}$.

Ainsi, Toland élabore-t-il dans le Tetradymus le projet d'une communauté idéale et restreinte, celle des sages recherchant la vérité sur toute chose. Il retrouve le souhait émis par Spinoza à la fin du livre cinq de L'Éthique concernant la communauté des sages dans la connaissance du troisième genre ${ }^{16}$. La vraie communauté comme communauté de philosophes déborde la définition même de toute communauté puisque l'appartenance sociale à un groupe en tant que telle est refusée au profit de la seule exigence de la connaissance vraie.

14. Ibid., p. 2 : «We all partake but too much of the Inclinations of those that give us Life, and of the Passions that are predominant in the Blood of the Family. "

15. J. Toland, « Clidophorus or of the Exoteric and Esoteric Philosophy », in Tetradymus, Londres, 1720, p. 70 : «From the senses arises Opinion, from reason Demonstration: on the former are huddled up the prejudices of the vulgar, following the bare appearance of things; on the latter are founded the axioms of the wise, who consider things as they are in themselves. "

16. Voir Alexandre Matheron, Individu et Communauté chez Spinoza, Paris, Minuit, 1969, quatrième partie, p. 594 : «l'unification interne : individualité libérée et communauté des sages. » Notre amour intellectuel de Dieu doit nous permettre de communiquer avec autrui de l'intérieur pour former une communauté de connaissance basée sur la complicité. 
Les quatrième et cinquième Lettres à Serena que Toland qualifie de philosophiques dans sa préface du texte, renvoient à la nécessité de la communauté philosophique dans la mesure où elles s'appliquent à une approche de la nature non selon l'opinion mais selon la connaissance, ce qui suppose une communauté de philosophes pour les lire, les accepter et les comprendre. Ce projet d'une communauté idéale dont l'utilité est tout d'abord de fournir une raison d'exister aux véritables textes de philosophie, confirme et exaspère le décalage entre une communauté réelle discréditée et une philosophie théorique de la nature. N'est philosophique chez Toland que ce qui renvoie à l'horizon d'un savoir, le lien social en général étant ferment d'erreurs et d'illusions qui obligent à élaborer une stratégie de l'écriture savante.

Si on y regarde de près, l'Enquête sur la vertu ou le mérite relève d'une tout autre conception de la nature que les quatrième et cinquième Lettres à Serena. Le titre même de l'ouvrage de Shaftesbury ne renvoie pas à une enquête sur la nature mais consiste en une recherche de ce qui est moral pour toute créature naturelle. En outre, tout ce qui se rapporte à la vertu constitue ce qu'il y a de plus moral mais aussi de plus naturel dans le monde, la vraie nature du monde n'étant pas extérieure et matérielle mais intérieure et morale. Le naturel est plus que la nature comme ensemble de corps matériels puisqu'il renvoie, comme nous l'avons souligné en introduction, à un « déisme humaniste » dans lequel le gouvernement de l'univers se fait en vue du bien. Le devenir moral du monde prouve la déité ${ }^{17}$.

De fait, contrairement à Toland, la vérité du monde ne consiste plus en une philosophie de la nature à visée théorique. L'organisation du monde fait l'économie d'une approche physique ou matérielle de la nature qui ne retient que la cause la plus immédiate de l'organisation du monde, l'activité des corps. Shaftesbury renoue avec un a priori dont la nature est prisonnière : l'activité de l'univers se fait en fonction d'un bien général

Diderot, traducteur de l'Enquête en 1745, souligne cette différence entre Shaftesbury et Toland puisqu'il oppose la conception de la déité chez Shaftesbury qui passe par le «théisme » aux déismes de Tindal et de Toland ${ }^{18}$.

17. Nous sommes d'accord avec Leslie STEPHEN, dans History of English Thought in the Eighteenth Century, repr. de l'éd. de Londres, 1902, Bristol, Thoemmes, 1991, vol. 2, p. 25 : pour Shaftesbury, dans l'Enquête, c'est la morale qui est ensuite théologique et métaphysique et non l'inverse.

18. Diderot conçoit cette différence à partir d'un rapport plus étroit du théisme au christianisme, le déisme étant plutôt à rapprocher de l'athéisme, du spinozisme. Cf. ShaftesBury, Essai de M.S*** sur le mérite et la vertu, «Discours préliminaire » de Denis DiDERor, 
Pour Diderot, qui fait référence à un passage précis des Moralistes $^{19}$, le théisme ne renie pas totalement la Révélation contrairement au déisme. Qu'est-ce à dire et en quoi cette précision peut-elle servir à notre propos? Le théisme n'exclut pas la Révélation, ce qui ne veut pas dire qu'il l'approuve mais, constituant pour Shaftesbury l'origine de toute religion, le véritable esprit du christianisme, il n'a pas de raison de la combattre comme le fait l'athéisme. La Révélation représente une dérivation historique de la religion pure ou naturelle.

La différence introduite par Diderot entre déisme et théisme, moins nette dans les textes de Shaftesbury que dans l'interprétation du philosophe français, recouvre une fois de plus la distinction d'Aldridge entre déismes humaniste et scientifique dans la mesure où représenter l'esprit du christianisme, c'est sans doute privilégier une approche religieuse ou encore humanisée, moralisée et non pas physique, matérielle de la nature :

«Croire que tout est gouverné, ordonné ou fait pour le mieux par un seul principe ou esprit essentiellement bon et permanent, c'est être un parfait théiste $»^{20}$.

L'ordonnancement du monde dans le théisme se fait au nom du lien d'amour, d'entente, de sympathie entre les créatures - relation mutuelle faisant taire les dissonances pour unir dans une sensation ou sentiment --, ce qu'il faut bien appeler le bien et, pour les créatures humaines, douées à la fois des sens et de la raison, la vertu.

Si la nature est vouée au positivisme et à la méthode scientifique pour un certain déisme, elle perd toute autonomie dans le théisme de Shaftesbury qui conçoit l'univers par un principe d'union et d'interdépendance de tous les éléments, dont le sens ultime est de tendre vers l'expression du bien : le lien entre les créatures est indissociablement naturel et social ou naturel parce que moral.

L'idée de nature n'a de sens qu'eu égard aux raisons morales de son existence qui donnent une dimension intérieure aux phénomènes naturels extérieurs. Cependant, c'est par l'être humain comme communauté ayant pour horizon commun la vertu que le monde est le plus conforme à sa nature.

Genève, 1769, p. 15 : « Je ne conçois pas comment, après des protestations aussi solennelles d'une entière soumission de cœur et d'esprit aux mystères sacrés de la religion, il s'est trouvé quelqu'un assez injuste pour compter M... S... au nombre des Asgil, des Tindal, et des Toland. "

19. Shaftesbury, op. cit. supra n. 4, vol. II, p. $209:$ "For as averse as I am to the Cause of Theism, or Name of Deist, when taken in a sense exclusive of Revelation; I consider still that, in strictness, the Root of all is Theism; and that to be a settled Christian, it is necessary to be first of all a good Theist. "

20. Ibid., p. 11 . 
Tout d'abord, la communauté humaine est une communauté de nature. Le lien social lorsqu'il est basé sur la sociabilité, disposition à sympathiser avec autrui, est constitutif, d'une part, de la définition même de la nature humaine et ressemble, d'autre part, au lien entre les êtres naturels dans le monde.

Ensuite, la communauté humaine est la communauté la plus naturelle car, avec elle, la sociabilité prend un sens véritablement moral comme participation à l'intérêt public et non réalisation des intérêts privés. En quoi l'impératif communautaire dans la société humaine inclut-il une continuité avec le monde de la nature?

De fait, le naturel n'existe que par l'impératif communautaire de l'être ensemble qui se réalise le plus pleinement dans la société faisant du lien social, comme disposition à la sociabilité, la manifestation la plus juste de ce naturel. Le modèle le plus achevé de l'organisation du monde est la communauté humaine. Comme principe d'explication de l'administration unitaire du monde, la composition des dispositions remplace la composition des forces physiques chez Toland, faisant du naturel non plus un objet d'étude théorique mais pratique. La véritable nature est dans la nature humaine comme nature morale.

La parfaite identification de la nature à l'impératif communautaire, dépend d'une maxime fondamentale dans la philosophie de Shaftesbury : le lien social comme lien sociable doit être préféré à la connaissance, alors que pour Toland la connaissance est la raison d'être de la vraie philosophie ésotérique. La compréhension du concept de nature à partir de l'être ensemble des créatures naturelles et, plus précisément, à partir du lien de communauté entre les hommes, rend problématique la connaissance de la nature dans une perspective proprement cosmologique et instaure le primat, dans la philosophie, de la notion de relation entre les hommes.

Shaftesbury se situe dans la tradition cicéronienne du Traité des devoirs ${ }^{21}$ lorsque Cicéron affirme la priorité des devoirs provenant des rapports sociaux sur ceux qui visent à la connaissance. Le devoir qui se rattache au lien social est le plus grand des devoirs. Dès lors, une communauté de sages n'est pas un regroupement d'érudits, de philosophes, mais c'est une communauté qui a pour objet de connaissance, la société réelle comme ensemble de rapports.

Pour conclure, nous pouvons remarquer que Toland élabore une véritable philosophie de la nature mais discrédite tout ce qu'une communauté a

21. Cicéron, Traité des devoirs, I, 43, in Les Stoiciens, Paris, Gallimard, « Bibliothèque de la Pléiade », 1962, p. 152-154 : «Les devoirs qui dérivent des rapports sociaux sont plus conformes à la nature que ceux qui visent à la connaissance. » 
d'arbitraire, de contingent au profit d'une société idéale pour laquelle le terme de communauté devient lui-même problématique.

En revanche, bien que Shaftesbury s'intéresse à la vérité du lien social en général pour analyser la société de manière dynamique, l'intégration du naturel dans le social ne risque-t-elle pas d'empêcher de penser radicalement le statut du nouveau, de l'imprévisible dans les relations humaines?

Fabienne BrUGERE (mai 1994). 